INPLASY

PROTOCOL

To cite: Liu et al. Efficacy and safety of Tuina for senile insomnia A protocol for systematic review and metaanalysis. Inplasy protocol 2021110063. doi:

10.37766/inplasy2021.11.0063

Received: 17 November 2021

Published: 17 November 2021

Corresponding author:

Yangshengjie Liu

liuyangshengjie@126.com

Author Affiliation:

Changchun University of

Chinese Medicine.

Support: Natural Science

Foundation.

Review Stage at time of this submission: The review has not yet started.

Conflicts of interest:

None declared.

\section{Efficacy and safety of Tuina for senile insomnia A protocol for systematic review and meta-analysis}

Liu, Y1; Liu, P22.

Review question / Objective: We conduct this study to evaluate the efficacy and safety of Tuina in the treatment of senile insomnia.

Condition being studied: Senile insomnia seriously affects the quality of life of the elderly. With the increase of the proportion of insomnia in the elderly, compared with the elderly with normal sleep quality, the elderly with long-term insomnia are more likely to have dizziness, fatigue, and decreased immunity. Tuina has shown good effects in the treatment of insomnia. At present, there is a lack of systematic review on acupuncture in the treatment of senile insomnia.

INPLASY registration number: This protocol was registered with the International Platform of Registered Systematic Review and Meta-Analysis Protocols (INPLASY) on 17 November 2021 and was last updated on 17 November 2021 (registration number INPLASY2021110063).

\section{INTRODUCTION}

Review question / Objective: We conduct this study to evaluate the efficacy and safety of Tuina in the treatment of senile insomnia.
Condition being studied: Senile insomnia seriously affects the quality of life of the elderly. With the increase of the proportion of insomnia in the elderly, compared with the elderly with normal sleep quality, the elderly with long-term insomnia are more 
likely to have dizziness, fatigue, and decreased immunity. Tuina has shown good effects in the treatment of insomnia. At present, there is a lack of systematic review on acupuncture in the treatment of senile insomnia.

\section{METHODS}

Participant or population: Patients ( $\geq 60$ years) with senile insomnia regardless of any race and gender.

Intervention: Tuina.

Comparator: Therapies other than Tuina (medicine, Chinese medicine, acupuncture, external therapy).

Study designs to be included: Patients ( $\geq 60$ years) with senile insomnia regardless of any race and gender; RCTs of Tuina for senile insomnia which were published in English and Chinese will be included; in the treatment group, Tuina was used alone or combined with other therapies, while in the control group, Tuina was not included; primary outcome: Pittsburgh sleep quality index, the insomnia severity index.

Information sources: We will search Chinese and English databases: China National Knowledge Infrastructure, Chinese Scientific and Journal Database,Wan Fang database (Wan fang), Chinese Biomedical LiteratureDatabase, PubMed, EMBASE, Cochrane library to identify articles of RCTs of Tuina for senile insomnia.

Main outcome(s): PSQI.

Additional outcome(s): SF-36, SAS, SDS.

Quality assessment / Risk of bias analysis: We will use the Standard 12 test to assess the statistical heterogeneity, $12<50 \%$ indicates insignificant, when $12 \geq 50 \%$ indicates significant. We will use a funnel plot to assess the reporting bias.

Strategy of data synthesis: We will use Revman 5.3 software for meta-analysis. We will userelative risk as the effective index for the count data, mean difference will be used for the measurement data as the effective index. The confidence interval of each effect index was set to $95 \%$. At the same time, the heterogeneity will be quantitatively assessed with 12 . If there was no statistical heterogeneity among the studies, the fixed-effect model will be used for meta-analysis. If it is heterogeneous, the random-effects model will be used $\mathrm{P}<.05$ indicates statistical significance.

Subgroup analysis: When there is some potential heterogeneity in this study, we may conduct subgroup analysis according to the genders, different ages, and different treatment times of included participants, if all the information could be available from included studies.

Sensitivity analysis: Sensitivity analysis will be conducted for assessing the robustness of the included results. Studies of high-risk bias will be excluded if the results are unstable.

Country(ies) involved: China.

Keywords: Tuina, senile insomnia.

Contributions of each author:

Author 1 - Yangshengjie Liu.

Author 2 - Peng Liu. 